\title{
Chemoprevention of Schistosomiasis: In vitro Antiparasitic Activity of Nineteen Plant-derived and Synthetic Simple Naphthoquinones and Naphthols against Schistosoma Mansoni Adult Worms
}

\author{
Lizandra G. Magalhães ${ }^{1 *}$, G. Subba Rao ${ }^{2}$, Ingrid A. O. Soares ${ }^{1}$, Fernanda R. Badocco ${ }^{1}$, Wilson R. Cunha ${ }^{1}$, Vanderlei Rodrigues ${ }^{3}$, and
} Govind J. Kapadia ${ }^{4}$

${ }^{1}$ Research Group on Natural Products, Center for Research in Sciences and Technology, University of Franca, Franca, Brazil ${ }^{2}$ Global Biotechnology Resource Center, 145 Rosewood Drive, Streamwood, IL 60107, USA

${ }^{3}$ Department of Biochemistry and Immunology, University of São Paulo, Medicine School of Ribeirão Preto, Ribeirão Preto, Brazil

${ }^{4}$ Department of Pharmaceutical Sciences, College of Pharmacy, Howard University, Washington, DC 20059, USA

\begin{abstract}
Schistosomiasis, a debilitating disease dating back to ancient times, is currently endemic in 78 tropical and subtropical countries with 243 million people requiring treatment. Current treatment of schistosomiasis depends primarily on a single drug, praziquantel which is less effective against larval stage of the parasite and has potential for development of resistance. Thus, there is urgent need for development of new, effective and inexpensive antischistosomal drugs. Simple naphthoquinone (NAPQ) secondary metabolites in plants are known to act as phytotoxins in preventing bacterial, fungal and parasitic attacks. The present study reports antischistosomal activity of nineteen plant-derived and synthetic simple NAPQs and naphthols against Schistosoma mansoni adult worms under in vitro conditions. Four of the tested compounds met the WHO's Special Program for Research and Training in Tropical Diseases (TDR) in vitro criterion for "hit" and lead compound (100\% mortality of adult worms at a concentration of $\leq 5 \mu \mathrm{g} / \mathrm{ml}$ when incubated for $48 \mathrm{~h}$ ): plant-derived naphthazarin, two synthetic NAPQs, 1, 4-NAPQ and 2-methy-1, 4-NAPQ (menadione) and synthetic 1-amino-2-naphthol hydrochloride. Structure-antischistosomal activity studies with 1, 4-NAPQs indicated the importance of the number and position of hydroxyl and methyl groups, particularly at C-2, C-5 and C-8 positions of the parent NAPQ molecule, which play important role in stabilizing quinone moiety and formation of reactive oxygen species essential for antiparasitic effect. The results call for further in vivo studies on the chemopreventive potential of plant-derived naphthazarin and synthetic 1,4-NAPQ, menadione and 1-amino-2-naphthol, all of which have met the WHO/TDR in vitro criterion for their consideration as lead schistosomicidal candidates.
\end{abstract}

Keywords: Schistosoma mansoni; 1, 2- and 1, 4-naphthoquinones; Naphthols; Chemoprevention

\section{Introduction}

Schistosomiasis is a tropical disease caused by trematodes of the genus Schistosoma [1] and World Health Organization (WHO) considers this water-borne, communicable disease a major public health problem of the 21st century [2]. While an estimated 779 million people are at risk worldwide [3], in 2011 at least 243 million people required treatment for this parasitic disease in 78 tropical and subtropical countries, including China. Schistosomiasis has been the scourge of humanity since ancient times. There is evidence that it was prevalent in ancient Egypt as early as 1250-1000 BC by the discovery of calcified schistosomes eggs in the kidneys of mummies [4]. Similar discoveries have been made with Chinese mummies dating back to $400 \mathrm{BC}$ [5]. It was first described as a tropical parasitic disease by the German physician Theodore Maximillian Bilharz in 1851 while working in a Cairo hospital [6]. Oliver Tracy Logan, an American physician, reported the first clinical case of schistosomiasis in Hunan province of China in 1905 [7]. Bilharz's pioneering studies traced the origin of this chronic infection to the larval form of Schistosoma parasite released by fresh water snails which act as the intermediate host and cause infection in humans by penetrating skin when it comes in contact with infested water used for swimming and bathing. In the human body, the larvae develop into adult schistosomes and the females release eggs in the blood vessels. Symptoms of the disease are caused by the body's reaction to the eggs and not by the worms themselves [2]. Adult S. mansoni parasites can survive in the mesenteric veins and enter the hepatic portal circulation system for up to 30 years without being eliminated by the immune attack in their human host [8].

People are at risk of schistosomiasis infection (also known as bilharzia, named after its discoverer) mostly in developing countries due to domestic, recreational and agricultural activities which expose them to infested water. The adverse economic and health consequences of this disease are considerable. In tropical countries, it is the second most socioeconomically devastating parasitic disease after malaria. In children it can cause anemia, stunting and learning disability, although they can be reversed by prompt treatment. Chronic schistosomiasis in adults can affect their ability to work and lead to infertility which is irreversible. Urogenital schistosomiasis results in kidney damage and fibrosis of the bladder and ureter, and if untreated, could advance to cancers. More than 200,000 deaths per year in sub-Saharan Africa are attributable to chronic schistosomiasis [2].

*Corresponding author: Lizandra Guidi Magalhães, Research Group on Natural Products, Center for Research in Sciences and Technology, University of Franca, Franca, 14404 600, São Paulo, Brazil; Tel: +55-16-3711-8878; E-mail: lizandra.magalhaes@unifran.edu.br

Received July 20, 2014; Accepted July 23, 2014; Published August 12, 2014

Citation: Magalhães LG, Rao GS, Soares IAO, Badocco FR, Cunha WR, et al. (2014) Chemoprevention of Schistosomiasis: In vitro Antiparasitic Activity of Nineteen Plant-derived and Synthetic Simple Naphthoquinones and Naphthols against Schistosoma Mansoni Adult Worms. J Trop Dis 2: 146. doi: 10.4172/2329891X.1000146

Copyright: (C 2014 Magalhães LG, et al. This is an open-access article distributed under the terms of the Creative Commons Attribution License, which permits unrestricted use, distribution, and reproduction in any medium, provided the original author and source are credited. 
Citation: Magalhães LG, Rao GS, Soares IAO, Badocco FR, Cunha WR, et al. (2014) Chemoprevention of Schistosomiasis: In vitro Antiparasitic Activity of Nineteen Plant-derived and Synthetic Simple Naphthoquinones and Naphthols against Schistosoma Mansoni Adult Worms. J Trop Dis 2: 146. doi: 10.4172/2329-891X.1000146

Despite recent advances in treatment and control of schistosomiasis, over 700 million people live in areas considered endemic in 78 countries including Africa, South America and China, and it continues to spread to new geographic regions $[2,9]$. Bilharz identified the genus Schistosoma among which S. mansoni is the most prevalent species infecting millions in Africa, the Middle East, the Caribbean, Brazil, Suriname and Venezuela. Currently, praziquantel (PZQ), first introduced in 1975, is the drug of choice for treatment of $S$. mansoni infection with oxamniquine as an infrequently used alternative for adults [10]. However, both drugs have limited effect on juvenile schistosomes and on already developed liver and spleen lesions [11]. Additionally, development of resistance to these drugs is a major concern $[12,13]$. Schistosomiasis treatment will reach crisis state if parasites acquire resistance to PZQ. Thus, current reliance on essentially a single drug in controlling such a widespread disease as schistosomiasis has raised alarm among medical practitioners and scientific investigators [9]. There is consensus on urgent need for research in developing new and affordable drugs against this debilitating parasitic disease which is considered a neglected infection primarily affecting developing countries.

Plants produce variety of secondary metabolites among which simple naphthoquinones (NAPQs) are known to act as phytotoxins to ward off bacterial, fungal and parasitic attacks [14]. Recent studies have found several NAPQs of plant origin to be potent antibacterial, antifungal and cytotoxic chemopreventive agents [15-19]. Also, antiparasitic activity of the following plant-derived NAPQs have been reported: lapachol commonly occurring in Bignoniaceae plants [20] and plumbagin [21] as leishmanicidal; plumbagin and 2-methylnaphthazarin from Nepenthes thorelii as antimalarials [22,23]; plumbagin against Fasciola gigantica [24]; juglone against Hymenolepes nana [25] and lapachol against Trypanosoma cruzi [26]. Earlier studies have shown antiparasitic effect of synthetic NAPQs, such as menadione on S. mansoni [27] and blockage of cercarial skin penetration by NAPQ derivatives [28]. Recently, in vitro anthelmintic effect of plant-derived NAPQs, plumbagin against $S$. mansoni adult worms [29,30] and lapachol potassium salt against $S$. mansoni cercariae [31] have been reported.

In continuation of our investigations of plant-derived and related synthetic antiparasitic compounds [32-34], we have studied nineteen plant-derived and synthetic simple NAPQs and naphthols derivatives (Figure 1) against S. mansoni adult worms under in vitro conditions. The objectives of the present study included comparison of their chemical structure and available antioxidation potential data with antischistosomal activity.

\section{Material and Methods}

\section{Chemicals}

The following chemicals were purchased from Aldrich Chemi-cal Company, Milwaukee, WI, USA: 5-hydroxy-1,4naphthoquinone (lawsone) (NAP-1); 5,8-dihydroxy-1,4naphthoquinone (naphthaza-rin) (NAP-2); 5-hydroxyl-1,4naphthoquinone (juglone) (NAP-3); 2-hydroxy-3-(3-methylbut-2enyl)-1,4-naphthoquinone (lapachol) (NAP-4); 1,4naphthoquinone (NAP-5); 2-methy1,4-naphthoquinone (menadione, vitamin K3) (NAP-6); 2-amino-3-chloro-1,4naphthoqui-none (herbicide ACNQ) (NAP-7); 2,3-dichloro-1,4napahthoquinone (NAP-8); 2,3-dibromo -1,4-naphthoquinone (NAP-9); 2,3-dichlo-ro-5,8-dihydroxy-1,4-naphthoquinone (NAP-10); bis-lawsone (NAP- 12); 1,2-naphthoquinone (NAP-13); 1,2-naphthoquinone-4-sulphonoc acid sodium salt (NAP-15); 1-
Plant-derived 1,4 napthoquinones<smiles>O=C1CC(O)C(=O)c2ccccc21</smiles><smiles>O=C1C=CC(=O)c2c(O)ccc(O)c21</smiles><smiles>O=C1C=CC(=O)c2c(O)cccc21</smiles><smiles>CC1=CC(=O)c2c(O)cccc2C1=O</smiles><smiles>CC(C)=CCC1=C(O)C(=O)c2ccccc2C1=O</smiles>

Plant-derived 1,4 \& 1,2-napthoquinones

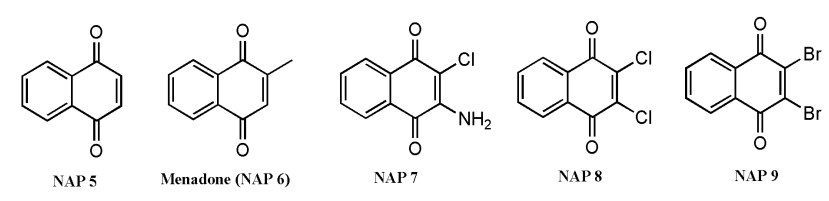<smiles>O=C1C(=O)c2c(O)ccc(O)c2C(=O)C(Cl)=C1Cl</smiles><smiles>Nc1ccc(O)c2ccccc12</smiles>

Figure 1: Chemical structures of plant-derived and synthetic naphthoquinones and naphthols tested for antiparasitic activity against Schistosoma mansoni adult worms.

amino-2-naphthol hydrochloride (NAP- 16); 1-amino-4-naphthol hydrochloride (NAP-17) 1,4-dihydroxy- 2-naphthoic acid (NAP-18); praziquantel (PZQ) and dimethyl sulfoxide (DMSO). 2,6Dihydroxynaphthalene (NAP-19) was procured from TCI America, Portland, OR, USA. Methylene-bis-lawsone (NAP-11) and 4amino-1, 2-naphthoquinone (NAP-14) were synthesized as reported earlier $[35,36]$.

\section{Animals and parasites}

The life cycle of the S. mansoni strain Luis Evangelista (LE) is maintained by passage through Biophalaria glabrata snails and $\mathrm{Balb} / \mathrm{c}$ mice at the Parasitology Research Laboratory, the University of Franca. This maintenance is authorized by the Ethical Committee for Animal Care of the University of Franca (Protocol: 028/12) in accordance with the internationally accepted principles for laboratory animal handling and care. Female mice (Balb/c weight $20-22 \mathrm{~g}$ ) were infected with 200 cercariae and after $56 \pm 2$ days of infection, $S$. mansoni adult worms were recovered under aseptic conditions by perfusion of the livers and mesenteric veins [37]. The worms were washed in RPMI 1640 medium (Gibco Life Technologies, Gaithersburg, MD, USA), kept at pH 7.5 with HEPES mM buffer, and supplemented with penicillin 
Citation: Magalhães LG, Rao GS, Soares IAO, Badocco FR, Cunha WR, et al. (2014) Chemoprevention of Schistosomiasis: In vitro Antiparasitic Activity of Nineteen Plant-derived and Synthetic Simple Naphthoquinones and Naphthols against Schistosoma Mansoni Adult Worms. J Trop Dis 2: 146. doi: 10.4172/2329-891X.1000146

Page 3 of 7

(100 UI/ml), streptomycin $(100 \mu \mathrm{g} / \mathrm{ml})$ and $10 \%$ bovine fetal serum (Gibco).

\section{In vitro studies with $S$. mansoni adult worms}

The procedure used has been described in detail earlier [33]. Briefly, one adult $S$. mansoni worm pair (one male and one female) in RPMI 1640 medium were placed in each of 24 -well plates. A preliminary screening of test compounds was performed at a concentration of $100 \mu \mathrm{M}$. The compounds were prepared in DMSO and added to the RPMI 1640 medium containing the worms after a period of $24 \mathrm{~h}$ of adaptation to the culture medium. The parasites were kept for $72 \mathrm{~h}$ in a constant temperature incubator at $37^{\circ} \mathrm{C}$ in an atmosphere of $5 \% \mathrm{CO}$ and monitored every $24 \mathrm{~h}$ for motor activity and mortality. Alteration in motor activity was classified as either slight, defined as a reduction in movement compared with the negative control or significant, defined as minimal movement observed for $1 \mathrm{~min}$. The worms we re considered dead when no movement was observed for at least $2 \mathrm{~min}$ [38]. The test compounds that killed all parasites (100\%) during 24, 48 or $72 \mathrm{~h}$ incubation periods were further evaluated at concentrations of $6.125 \mu \mathrm{M}$ to $100 \mu \mathrm{M}$. The minimum lethal concentration (MLC) which is the minimum concentration needed to kill all worms and the 50\% lethal concentration (LC50) which is the concentration that kills $50 \%$ of worms for these test compounds were determined after incubating for $24 \mathrm{~h}, 48 \mathrm{~h}$ and $72 \mathrm{~h}$ [39]. The LC50 values were calculated from nonlinear regression dose-response mortality graphs. All experiments were carried out in quadruplicate ( 8 worms in each experiment) and repeated two times (total of 16 worms). Adult worms incubated with the highest concentration of solvent $(0.1 \%$ DMSO $)$ served as negative control while those incubated with PZQ $(12.5 \mu \mathrm{M})$ served as positive control.

\section{Statistical analysis}

Results are expressed as the mean \pm S.D. The statistical tests were performed with the Graphpad Prism (version 5.0) software. Data were statistically analyzed by one-way analysis of variance, followed by Dunnet's comparison.

\section{Results}

In the preliminary screening, the nineteen plant-derived and synthetic NAPQ and naphthol compounds (Figure 1) were tested at $100 \mu \mathrm{M}$ concentration and the following ten compounds $(52.6 \%$ of compounds tested) were found to cause death of $100 \%$ of adult $S$. mansoni worms when incubated for $24 \mathrm{~h}$ (Table 1): two plant-derived 1,4-NAPQs, naphthazarin (NAP-2) and juglone (NAP-3); synthetic analogs, five 1,4-NAPQs (NAP-5, NAP-6, NAP-8, NAP-9 and NAP10); 1,2-NAPQ-4-sulfonic acid (NAP-15) and two 1-amino-naphthols (NAP-16 and NAP-17). Among the synthetic NAPQs, 1,2-NAPQ (NAP-13) caused $100 \%$ mortality of worms when incubated for 48 and $72 \mathrm{~h}$, while herbicide ACNQ (NAP-7) and methylene-bis-lawsone (NAP-11) caused $100 \%$ mortality of parasites only when incubated for $72 \mathrm{~h}$. A $25 \%$ reduction in mortality of adult schistosomes was observed with NAP-7 at $24 \mathrm{~h}$ which increased to $50 \%$ at $48 \mathrm{~h}$ of incubation. With synthetic 4-amino-1, 2-NAPQ, (NAP-14) and naphthol carboxylic acid (NAP- 18), worm mortality remained at $25 \%$ when incubated for 48 or $72 \mathrm{~h}$ with no lethal effect observed at 24-h incubation period. Also, there was no difference in mortality rate between male and female $S$. mansoni adult worms.

Among the plant-derived 1, 4-NAPQs studied, lawsone (NAP-1) and lapachol (NAP-4), and the synthetic bis-lawsone (NAP-12) and 2, 6-naphthol (NAP-19) were inactive ( $0 \%$ mortality) against S. mansoni

\begin{tabular}{|c|c|c|c|}
\hline \multirow{2}{*}{ Compound } & \multicolumn{3}{|c|}{ Mortality \pm SDa $(\%)$} \\
\hline & $24 \mathrm{~h}$ & $48 \mathrm{~h}$ & $72 \mathrm{~h}$ \\
\hline Negative control (DMSO) & $0 \pm 0.00$ & $0 \pm 0.00$ & $0 \pm 0.00$ \\
\hline Positive control (PZQ) & $100 \pm 0.00^{d}$ & $100 \pm 0.00^{d}$ & $100 \pm 0.00^{d}$ \\
\hline \multicolumn{4}{|l|}{$\begin{array}{l}\text { Plant-derived } \\
\text { 1,4-naphthoquinones }\end{array}$} \\
\hline NAP-1 (Lawsone) & $0 \pm 0.00$ & $0 \pm 0.00$ & $0 \pm 0.00$ \\
\hline NAP-2 (Naphthazarin) & $100 \pm 0.00^{d}$ & $100 \pm 0.00^{d}$ & $100 \pm 0.00^{d}$ \\
\hline NAP-3 (Juglone) & $100 \pm 0.00^{d}$ & $100 \pm 0.00^{d}$ & $100 \pm 0.00^{d}$ \\
\hline NAP-4 (Lapachol) & $0 \pm 0.00$ & $0 \pm 0.00$ & $0 \pm 0.00$ \\
\hline \multicolumn{4}{|l|}{$\begin{array}{l}\text { Synthetic } 1,4 \text { - and } \\
\text { 1,2-naphthoquinones }\end{array}$} \\
\hline NAP-5 & $100 \pm 0.00^{d}$ & $100 \pm 0.00^{\mathrm{d}}$ & $100 \pm 0.00^{d}$ \\
\hline NAP-6 (Menadione) & $100 \pm 0.00^{d}$ & $100 \pm 0.00^{d}$ & $100 \pm 0.00^{d}$ \\
\hline NAP-7(ACNQ) & $25 \pm 17.68$ & $50 \pm 17.68^{d}$ & $100 \pm 0.00^{d}$ \\
\hline NAP-8 & $100 \pm 0.00^{d}$ & $100 \pm 0.00^{d}$ & $100 \pm 0.00^{d}$ \\
\hline NAP-9 & $100 \pm 0.00^{d}$ & $100 \pm 0.00^{d}$ & $100 \pm 0.00^{d}$ \\
\hline NAP-10 & $100 \pm 0.00^{d}$ & $100 \pm 0.00^{d}$ & $100 \pm 0.00^{d}$ \\
\hline NAP-11 & $50 \pm 17.68^{d}$ & $81 \pm 17.68^{d}$ & $100 \pm 0.00^{d}$ \\
\hline NAP-12 & $0 \pm 0.00$ & $0 \pm 0.00$ & $0 \pm 0.00$ \\
\hline NAP-13 & $0 \pm 0.00$ & $100 \pm 0.00^{d}$ & $100 \pm 0.00^{d}$ \\
\hline NAP-14 & $0 \pm 0.00$ & $25 \pm 17.68$ & $25 \pm 17.68$ \\
\hline NAP-15 & $100 \pm 0.00^{d}$ & $100 \pm 0.00^{d}$ & $100 \pm 0.00^{d}$ \\
\hline \multicolumn{4}{|l|}{ Synthetic naphthols } \\
\hline NAP-16 & $100 \pm 0.00^{d}$ & $100 \pm 0.00^{d}$ & $100 \pm 0.00^{d}$ \\
\hline NAP-17 & $100 \pm 0.00^{d}$ & $100 \pm 0.00^{d}$ & $100 \pm 0.00^{d}$ \\
\hline NAP-18 & $0 \pm 0.00$ & $25 \pm 17.68$ & $25 \pm 17.68$ \\
\hline NAP-19 & $0 \pm 0.00$ & $0 \pm 0.00$ & $0 \pm 0.00$ \\
\hline
\end{tabular}

aMean percentage $\pm S D$ of two independent experiments using 8 adult $S$. mansoni worms in each experiment incubated for $24-72 \mathrm{~h}$. Worms were considered dead when no movement was observed for at least $2 \mathrm{~min}$

${ }^{\mathrm{b} D M S O}$ solvent $(0.1 \%)+\mathrm{RPMI}$ medium

${ }^{\mathrm{C} P Z Q}$ (praziquantel) tested at a concentration of $12.5 \mu \mathrm{M}$

'Indicate statistically significant differences compared with negative control group $(P<0.001)$

Table 1: Effect of plant-derived and synthetic naphthoquinone and naphthol compounds at $100 \mu \mathrm{M}$ concentration on mortality of S. mansoni adult worms in vitro.

when tested at $100 \mu \mathrm{M}$ concentration for 24,48 and $72 \mathrm{~h}$ (Table 1). The synthetic naphthol carboxylic acid (NAP-18) and 4-amino-1, 2-NAPQ (NAP-14) were inactive when incubated with worms for 24 $\mathrm{h}$ but exhibited $25 \%$ mortality rate upon increasing incubation period to 48 and $72 \mathrm{~h}$. Decreased parasite motor activity was observed with synthetic 1, 2-NAPQ (NAP-13) when incubated for 48 and $72 \mathrm{~h}$, and with methylene-bis-lawsone (NAP-11) after 72-h incubation only. All compounds that did not cause death of $S$. mansoni adult worms at $100 \mu \mathrm{M}$ concentration (NAP-1, NAP-4, NAP-12, NAP-14, NAP-18 and NAP-19) when incubated for 24, 48 and/or $72 \mathrm{~h}$ showed decrease (25 to $100 \%$ ) in motor activity (Table 2 ). The positive control (PZQ at $12.5 \mu \mathrm{M}$ concentration) exhibited $100 \%$ mortality of worms after $24-\mathrm{h}$ incubation period while the negative control (DMSO solvent + RPMI medium) caused no parasite mortality at all incubation periods tested (24 to $72 \mathrm{~h}$ ) (Tables 1 and 2).

Among the plant-derived 1, 4-NAPQs, naphthazarin (NAP-2) was the most active antischistosomal compound with MLC of $25 \mu \mathrm{M}$ and LC50 of $20.33 \mu \mathrm{M}$ when tested for 24 -h incubation period. For similar incubation period of $24-\mathrm{h}$, the most active synthetic compounds were, in order of potency, 1-amino-2-naphthol hydrochloride (NAP-16), 1,4-NAPQs NAP-5 and NAP-10 with MLC of $25 \mu \mathrm{M}$ and LC50 of 11.77, 14.40 and $20.33 \mu \mathrm{M}$, respectively. Between 2- and 4-naphthols 
Citation: Magalhães LG, Rao GS, Soares IAO, Badocco FR, Cunha WR, et al. (2014) Chemoprevention of Schistosomiasis: In vitro Antiparasitic Activity of Nineteen Plant-derived and Synthetic Simple Naphthoquinones and Naphthols against Schistosoma Mansoni Adult Worms. J Trop Dis 2: 146. doi: 10.4172/2329-891X.1000146

Page 4 of 7

\begin{tabular}{|c|c|c|c|c|c|c|}
\hline \multirow{3}{*}{ Compound } & \multicolumn{6}{|c|}{ Motor Activity Reduction \pm SD $^{a}(\%)$} \\
\hline & \multicolumn{2}{|c|}{$24 \mathrm{~h}$} & \multicolumn{2}{|c|}{$48 \mathrm{~h}$} & \multicolumn{2}{|c|}{$72 \mathrm{~h}$} \\
\hline & Slight & Significant & Slight & Significant & Slight & \\
\hline Negative control (DMSO) ${ }^{\mathrm{b}}$ & $0 \pm 0.00$ & $0 \pm 0.00$ & $0 \pm 0.00$ & $0 \pm 0.00$ & $0 \pm 0.00$ & $0 \pm 0.00$ \\
\hline Positive control $(\mathrm{PZQ})^{\mathrm{c}}$ & - & - & - & - & - & - \\
\hline \multicolumn{7}{|c|}{ Plant-derived 1,4-naphthoquinones } \\
\hline NAP-1 (Lawsone) & $100 \pm 000^{d}$ & $0 \pm 0.00$ & $75 \pm 17.68^{d}$ & $25 \pm 17.68$ & $75 \pm 17.68^{d}$ & $25 \pm 17.68$ \\
\hline NAP-2 ( Naphthazarin) & - & - & - & - & - & - \\
\hline NAP-3 (Juglone) & - & - & - & - & - & - \\
\hline NAP-4 (Lapachol) & $75 \pm 17.68^{d}$ & $0 \pm 0.00$ & $75 \pm 17.68^{d}$ & $0 \pm 0.00$ & $100 \pm 0.00^{d}$ & $0 \pm 0.00$ \\
\hline \multicolumn{7}{|c|}{ Synthetic 1,4- and 1,2-naphthoquinones } \\
\hline NAP-5 & - & - & - & - & - & - \\
\hline NAP-6 (Menadione) & - & - & - & - & - & - \\
\hline NAP-7(ACNQ) & $25 \pm 17.68$ & $50 \pm 17.68^{d}$ & $0 \pm 0.00$ & $50 \pm 17.68^{d}$ & - & - \\
\hline NAP-8 & - & - & - & - & - & - \\
\hline NAP-9 & - & - & - & - & - & - \\
\hline NAP-10 & - & - & - & - & - & - \\
\hline NAP-11 & $0 \pm 0.00$ & $44 \pm 17.68^{d}$ & $0 \pm 0.00$ & $25 \pm 17.68^{d}$ & - & - \\
\hline NAP-12 & $100 \pm 0.00$ & $0 \pm 0.00$ & $100 \pm 0.00^{d}$ & $0 \pm 0.00$ & $100 \pm 0.00^{d}$ & $0 \pm 0.00$ \\
\hline NAP-13 & $0 \pm 0.00$ & $100 \pm 0.00^{d}$ & - & - & - & - \\
\hline NAP-14 & $50 \pm 26.52^{d}$ & $50 \pm 26.52^{d}$ & $25 \pm 17.68$ & $25 \pm 17.68$ & $0 \pm 0.00$ & $50 \pm 17.68^{d}$ \\
\hline NAP-15 & - & - & - & - & - & - \\
\hline \multicolumn{7}{|l|}{ Synthetic naphthols } \\
\hline NAP-16 & - & - & - & - & - & - \\
\hline NAP-17 & - & - & - & - & - & - \\
\hline NAP-18 & $25 \pm 17.68$ & $25 \pm 17.68$ & $25 \pm 17.68$ & $44 \pm 17.68^{d}$ & $25 \pm 17.68$ & $44 \pm 17.68^{d}$ \\
\hline NAP-19 & $25 \pm 17.68^{d}$ & $0 \pm 0.00$ & $25 \pm 17.68^{d}$ & $0 \pm 0.00$ & $25 \pm 17.68$ & $0 \pm 0.00$ \\
\hline
\end{tabular}

aMean percentage $\pm S D$ of two independent experiments using 8 adult $S$. mansoni worms in each experiment incubated for $24-72 \mathrm{~h}$. 'Slight' indicates a reduction in movement compared with the negative control and

'Significant' indicates minimal movement observed during 1 minute. $0=$ no reduction; 25 to 100 are percentage reductions and '-'indicates worms dead.

bDMSO solvent $(0.1 \%)+$ RPMI medium.

${ }^{\mathrm{c}} \mathrm{PZQ}$ (praziquantel) tested at a concentration of $12.5 \mu \mathrm{M}$.

dStatistically significant differences compared with negative control group $(P<0.001)$

Table 2: Effect of plant-derived and synthetic naphthoquinone and naphthol compounds at $100 \mu \mathrm{M}$ concentrations on motor activity reduction of $S$. mansoni adult worms in vitro.

with amino group at C-1 position, NAP-16 and NAP-17, the former derivative exhibited higher antiparasitic activity except at extended incubation periods of 48 and $72 \mathrm{~h}(\mathrm{LC} 50=10.04 \mathrm{vs} 11.77 \mu \mathrm{M})$. For 72-h incubation period, the synthetic 1,4-NAPQ menadione (NAP-6) was the most active among the nineteen compounds tested against $S$. mansoni in the present screening with MLC of $12.5 \mu \mathrm{M}$ and LC50 of $5.80 \mu \mathrm{M}$ (Table 3).

\section{Discussion}

On-going research on plant-derived simple NAPQs have demonstrated their remarkable antiparasitic, antimicrobial and cytotoxic properties, and provided lead for development of new synthetic analogs with higher potency and lower toxicity $[16,21]$. Recently, we reported potent antimalarial activity of a novel class of amino 1, 4NAPQs against Plasmodium falciparum [32] and leishmanicidal activity of halogenated 1, 4-NAPQs against Leishmania donovani [34]. The present investigation is a continuation of our research on antiparasitic activity of NAPQs which compares antischistosomal activity of nineteen plant-derived and synthetic simple NAPQs and naphthols (Figure 1) against $S$. mansoni adult worms under in vitro experimental conditions.

WHO's Special Program for Research and Training in Tropical Diseases (TDR) has outlined the following definition and activity criteria for hit and lead compound for the control of schistosomiasis: in vitro studies, $100 \%$ mortality of adult worms at a concentration of $\leq$ $5 \mu \mathrm{g} / \mathrm{ml}$ when incubated for $48 \mathrm{~h}$ and in vivo studies, $80 \%$ wohtrm reduction after five injections at a dose of $100 \mathrm{mg} / \mathrm{kg}$ body-weigt

\begin{tabular}{|l|c|c|c|c|c|c|}
\hline \multirow{2}{*}{ Compound } & \multicolumn{3}{|c|}{ MLC $(\boldsymbol{\mu M})$} & \multicolumn{3}{c|}{ LC $_{50}(\boldsymbol{\mu M})$} \\
\cline { 2 - 7 } & $24 \mathrm{~h}$ & $48 \mathrm{~h}$ & $72 \mathrm{~h}$ & $24 \mathrm{~h}$ & $48 \mathrm{~h}$ & $72 \mathrm{~h}$ \\
\hline PZQ $^{\mathbf{2}}$ & 3.12 & 3.12 & 1.56 & 0.86 & 0.86 & 0.78 \\
\hline $\begin{array}{l}\text { Plant-derived } \\
\text { 1,4-naphthoquinones }\end{array}$ & & & & & & \\
\hline NAP-2 (Naphthazarin) & 25.00 & 25.00 & 25.00 & 20.33 & 11.77 & 11.77 \\
\hline NAP-3 (Juglone) & 100.00 & 100.00 & 50.00 & 34.16 & 32.14 & 25.00 \\
\hline $\begin{array}{l}\text { Synthetic 1,4- and 1,2- } \\
\text { naphthoquinones }\end{array}$ & & & & & \\
\hline NAP-5 & 25.00 & 25.00 & 25.00 & 14.40 & 12.98 & 12.98 \\
\hline NAP-6(Menadione) & 50.00 & 25.00 & 12.50 & 11.77 & 10.64 & 5.80 \\
\hline NAP-7 (ACNQ) & $>100$ & $>100$ & 100.00 & 70.92 & 53.85 & 52.15 \\
\hline NAP-8 & 50.00 & 50.00 & 50.00 & 22.24 & 20.22 & 10.22 \\
\hline NAP-9 & 50.00 & 50.00 & 50.00 & 37.94 & 37.94 & 25.97 \\
\hline NAP-10 & 25.00 & 25.00 & 25.00 & 20.33 & 12.50 & 12.50 \\
\hline NAP-13 & 100.00 & 50.00 & 50.00 & 54.18 & 26.93 & 25.02 \\
\hline NAP-14 & $>100$ & $>100$ & 100.00 & 84.30 & 84.30 & 58.71 \\
\hline NAP-15 & 100.00 & 50.00 & 50.00 & 48.47 & 37.94 & 37.94 \\
\hline Synthetic naphthols & & & & & & \\
\hline NAP-16 & 25.00 & 25.00 & 25.00 & 11.77 & 11.77 & 11.77 \\
\hline NAP-17 & 100.00 & 50.00 & 50.00 & 20.15 & 10.04 & 10.04 \\
\hline
\end{tabular}

aMLC is the minimal lethal concentration needed to kill all 16 adult worms after 2448- or 72-h incubation period.

'LC50 is the lethal concentration needed to kill $50 \%$ of the adult worms after 24 48- or 72-h incubation period.

${ }^{\mathrm{P} Z Q Q}$ (praziquantel) positive control.

Table 3: Minimal lethal concentration (MLC)a and 50\% Lethal Concentration (LC50)b of plant-derived and synthetic naphthoquinone and naphthol compounds active against $S$. mansoni adult worms in vitro. 
Citation: Magalhães LG, Rao GS, Soares IAO, Badocco FR, Cunha WR, et al. (2014) Chemoprevention of Schistosomiasis: In vitro Antiparasitic Activity of Nineteen Plant-derived and Synthetic Simple Naphthoquinones and Naphthols against Schistosoma Mansoni Adult Worms. J Trop Dis 2: 146. doi: 10.4172/2329-891X.1000146

of test animal/day [30,40]. Among the nineteen plant-derived and synthetic simple NAPQs and naphthols tested in the present in vitro study with $S$. mansoni adult worms, four compounds met the $\mathrm{WHO} /$ TDR in vitro criterion for "hit" and lead compound: plant-derived naphthazarin (NAP-2), two synthetic 1,4-NAPQs (NAP-5 and NAP-6) and synthetic 1-amino-2-naphthol hydrochloride (NAP-16) with 100\% mortality of worms at $25 \mu \mathrm{M}$ concentration (equivalent to $4.75,3.95$, 4.30 and $4.9 \mu \mathrm{g} / \mathrm{ml}$, respectively) when incubated for $48 \mathrm{~h}$ (Table 3 ). These are recommended for further in vivo studies in animal models of schistosomiasis. Among the above four active compounds, menadione (NAP-6) is vitamin K3 which is a metabolite of orally ingested vitamin $\mathrm{K}[41]$ and found to be the third most active compound among the lead candidates that met WHO/TDR in vitro antiparasitic criteria against $S$. mansoni adult worms. It is noteworthy that in a recent study, the plant-derived plumbagin (Figure 1) has also met the above WHO/TDR criterion at $4.70 \mu \mathrm{g} / \mathrm{mL}$ concentration when incubated for $48 \mathrm{~h} \mathrm{[30]}$ and it is reported to be more active than the currently used antischistosomal drug PZQ under the in vitro experimental conditions employed [29].

Lawsone (NAP-1) and lapachol (NAP-4) among the plant-derived 1,4-NAPQs, and bis-lawsone (NAP-12) and 2,6-naphthol (NAP-19) among the synthetic compounds evaluated in this investigation were completely inactive ( $0 \%$ mortality) against $S$. mansoni when tested at $100 \mu \mathrm{M}$ concentration when incubated for 24,48 and $72 \mathrm{~h}$ (Table 1 ). In an earlier study [31], the potassium salt of lapachol (NAP-4) was found to be active against $S$. mansoni eggs with $\mathrm{LC}_{90}<3 \mu \mathrm{g} / \mathrm{ml}$ which may be attributable to higher solubility of the salt tested.

Although plant-derived 1,4-NAPQ juglone (NAP-3), synthetic halogenated 1,4-NAPQs (NAP-8, NAP-9 and NAP-10), 1-amino-4naphthol hydrochloride (NAP-17) and 1,2-NAPQ sulfonic acid sodium salt (NAP-15) caused $100 \%$ mortality of $S$. mansoni adult worms when incubated for 24, 48 and $72 \mathrm{~h}$ (Table 1), they failed to meet the WHO/ TDR criteria for consideration as "hit" and lead compound for further antischistosomal study (Table 3). Similarly, the remaining synthetic 1,4-NAPQs, herbicide ACNQ (NAP-7) and methylene-bis-lawsone (NAP-11), and 1,2-NAPQs (NAP-13 and NAP-14) and naphthol carboxylic acid (NAP- 18) did not meet the WHO/TDR criteria for further study against schistosomiasis.

Several structure-activity relationship (SAR) studies with NAPQs for identifying best candidates for treating parasitic diseases have been conducted earlier $[28,42]$. However, to-date no clinically viable antischistosomal compound has emerged from such endeavors. In the present investigation, comparison of chemical structures of active compounds against $S$. mansoni adult worms that met the WHO/TDR in vitro criterion for further antischistosomal study with those of lesser or no such activity (Figure 1 and Table 1)[30] indicated the dependence of antiparasitic activity on the number and nature of substituents in the parent NAPQ molecule. Thus, retention of antischistosomal activity with the addition of methyl group at C-2 position in the parent 1, 4-NAPQ molecule, as in menadione (NAP-6) and plumbagin, and hydroxyl group(s) at C-5 or C-5 and $\mathrm{C}-8$ positions, as in plumbagin and naphthazarin (NAP-2), was observed. This follows their reported NADPH-dependent oxidoreductase enzyme inhibitory activity (naphthazarin, $100 \%$, plumbagin $85 \%$ and menadione $82 \%$ ) [43]. However, there was substantial loss in antiparasitic activity when methyl group was absent at $\mathrm{C}-2$ position with hydroxyl group at $\mathrm{C}-5$ position, as in juglone (NAP-3) and complete loss of activity when hydroxyl group was present at C-2 position instead of methyl group, as in lawsone (NAP-1) and lapachol (NAP-4). This indicates the importance of location of hydroxyl (a strong electron donor) and methyl (a weak electron donor) groups in stabilizing the quinone moiety in the parent 1, 4-NAPQ structure. Such differential bioactivity of 1, 4-NPQs with the two functional groups, hydroxyl and methyl, at C-2/C-5 positions has also been observed with plumbagin (as both antimutagenic and antioxidant), juglone (antimutagenic only) and menadione (antioxidant only) when tested in the Ames mutagenic and DNA oxidative damage assays [44]. Other comparative studies with lawsone (NAP-1) and lapachol (NAP-4) have confirmed such inhibitory effect of hydroxyl group at C-2 position on antileishmanial activity [45] and pro-oxidant, cytochrome P450-linked monooxygenase systems [46].

The mechanism by which NAPQs exerts their in vitro schistosomicidal effect is not clear at the present time. However, it is generally recognized that mechanisms of antiparasitic activity involve modulation of parasite redox cycling (reduction and oxidation cycle of quinones in flavin enzymes such as NADPH-cytochrome P450 and mitochondrial NADH-ubiquinone oxidoreductase) and production of reactive oxygen species (ROS) leading to severe oxidative stress by oxidizing essential macromolecules, such as proteins, lipids and DNA $[47,48]$. Studies have shown that NAPQs are potent electrophiles and they are also capable of directly reacting with thiol groups of $S$. mansoni parasite proteins and glutathione resulting in inhibition of enzymes essential for parasite survival $[43,49]$. While NAPQs are capable of acting both as modulator of redox cycling and as electrophiles, one of these two mode of actions may play a dominant role in eliciting antiparasitic activity of individual compounds based on their chemical structure $[40,50,51]$.

In conclusion, the present in vitro antischistosomal studies with nineteen plant-derived and synthetic simple NAPQs and naphthols against $S$. mansoni adult worms identified plant-derived naphthazarin (NAP-2), synthetic NAPQs (NAP-5 and NAP-6) and 1-amino-2naphthol hydrochloride (NAP-16) as hit and lead compounds that met the $\mathrm{WHO} / \mathrm{TDR}$ in vitro criterion for further in vivo studies in animal models of schistosomiasis. Although several plant-derived and synthetic NAPQs tested in this study are known to exhibit diverse antiparasitic effects, the structural requirement for this group of antischistosomal compounds appears to be different from those required for their activity against other parasites afflicting humans. While this is the first report on the antiparasitic activity of synthetic amino derivatives of simple naphthols, their mode of action against schistosomes remains to be delineated. Based on the results of this in vitro investigation, further chemopreventive in vivo studies are called for on the plantderived naphthazarin (NAP-2) and synthetic menadione (NAP-6), 1,4NAPQ (NAP-5) and 1-amino-2-naphthol (NAP-16), all of which have met the $\mathrm{WHO} / \mathrm{TDR}$ in vitro criterion for their consideration as lead schistosomicidal candidates.

\section{Acknowledgements}

GJK thanks Dean Anthony Wutoh for continued support and Dr. Xionghao Lin for assistance in drawing of chemical structures. This research was supported in part by São Paulo Research Foundation, Brazil - FAPESP (Process: 2013/111644; 2014/02018-7), National Counsel of Technological and Scientific Development Brazil - CNPQ and the Howard University Fund for Academic Excellence.

\section{Conflict of Interest}

The authors declare that they have no conflict of interest.

\section{References}

1. Gray DJ, Ross AG, Li YS, McManus DP (2011) Diagnosis and management of schistosomiasis. Br Med J 342: d2651.

2. Schistosomiasis. Fact Sheet No. 115 (2014) World Health Organization Geneva, Switzerland. 
Citation: Magalhães LG, Rao GS, Soares IAO, Badocco FR, Cunha WR, et al. (2014) Chemoprevention of Schistosomiasis: In vitro Antiparasitic Activity of Nineteen Plant-derived and Synthetic Simple Naphthoquinones and Naphthols against Schistosoma Mansoni Adult Worms. J Trop Dis 2: 146. doi: 10.4172/2329-891X.1000146

3. Steinmann P, Keiser J, Bos R, Tanner M, Ultzinger J (2006) Schistosomiasis and water resources development: systematic review, meta-analysis, and estimates of people at risk. Lancet Infect Dis 6: 411-425.

4. Ruffer MA (1910) Note on the presence of Bilharzia haematobium in Egyptian mummies of the twentieth dynasty (1250-1000 BC). Br Med J 1: 16.

5. Berry-Caban CS (2007) Return of the God of Plague: Schistosomiasis in China. J Rural Trop Pub Health 6: 45-53.

6. El Tawil KM, Plassmann M (2011) Theodor Maximillian Bilharz (1825-1862): The discoverer of schistosomiasis. Int J Parasitol Res 3: 17-25.

7. Logan OT (1905) A case of dysentery in Hunan Province caused by the trematodes, Schistosoma japonicum. China Med Mission J 19: 243-245.

8. Vermund SH, Bradley DJ, Ruiz-Tiben E (1983) Survival of Schistosoma mansoni in the human host: Estimates from a community-based prospective study in Puerto Rico. Am J Trop Med Hyg 32: 1040-1048.

9. El Ridi RAF, Tallima HAM (2013) Novel therapeutic and preventive approaches for schistosomiasis: Review. J Adv Res 4: 467-478.

10. Danso-Appiah A, Olliaro PL, Donegan S, Sinclair D, Utzinger J (2013) Drugs for treating Schistosoma mansoni infection. Cochrane Database Syst Rev 2 CD000528.

11. Sabah AA, Fletcher C, Webbe G, Doenhoff MJ (1986) Schistosoma mansoni: chemotherapy of infections of different ages. Exp Parasitol 61: 294-303.

12. Doenhoff MJ, Kusel JR, Coles GC, Cioli D (2002) Resistance of Schistosoma mansoni to praziquantel: Is there a problem? Trans R Soc Trop Med Hyg 96 465-469.

13. Chai JY (2013) Praziquantel treatment in trematodes and cestode infections: an update. Infect Chemother 45: 42-43.

14. Babula P, Adam V, Havel L, Kizek R (2009) Noteworthy secondary metabolites naphthoquinones -their occurrence, pharmacological properties and analysis. Curr Pharmaceut Anal 5: 47-68.

15. Kapadia GJ, Balasubramanian V, Tokuda $\mathrm{H}$, Konoshima $\mathrm{T}$, Takasaki M, et al. (1997) Anti-tumor promoting effects of naphthoquinones derivatives on short-term Epstein-Barr virus early antigen activation assay and mouse skin carcinogenesis. Cancer Lett 113: 47-53.

16. Riffel A, Medina LF, Stefani V, Santos RC, Bizani D, et al. (2002) In vitro antimicrobial activity of a new series of 1,4-naphthoquinones. Braz J Med Biol Res 35: 811-818.

17. Eilenberg H, Pnini-Cohen S, Rahamin Y, Sionov E, Segal E, et al. (2010) Induced antifungal naphthoquinones in the pitchers of the carnivorous plant Nepenthes khasia. J Exp Bot 61: 911-922.

18. Sand JM, Hafeez BB, Jamal MS, Witkowsky O, Siebers EM, et al. (2012) Plumbagin (5-hydroxy-2-methyl-1,4-naphtahquinone) isolated from Plumbago zeylamica, inhibits ultraviolet radiation-induced development of squamous cell carcinomas. Carcinogenesis 33: 184-190.

19. Kapadia GJ, Rao GS, Sridhar R, Ichiishi E, Takasaki M, et al. (2013) Chemoprevention of skin cancer: Effect of Lawsonia inermis L. (Henna) lea powder and its pigment artifact, lawsone in the Epstein-Barr virus early antigen activation assay and in two-stage mouse skin carcinogenesis models. AntiCancer Agent Med Chem 13: 1500-15007.

20. Teixeira MJ, de Almeida YM, Viana JR, Holanda Filha JG, Rodrigues TP, et al.(2001) In vitro and in vivo leishmanicidal activity of 2-hydroxy-3-(3methyl-2-butenyl)-1,4-naphthoquinone (lapachol). Phytother Res 15: 44-48.

21. Kayser O, Kiderlen AF, Laatsch $\mathrm{H}$, Croft SL (2000) In vitro leishmanicida activity of monomeric and dimeric naphthoquinones. Acta Trop 77: 307-314.

22. Likhitwitayawuid K, Kaewamatawong R, Ruangrungsi N, Krungkrai J (1998) Antimalarial naphthoquinones from Nepenthus thorelii. Planta Med 64: 237

23. Sumsakul W, Plengsuriyakam T, Chaijaroenkul W, Viyanant V, Karbwang J et al. (2014) Antimalarial activity of plumbagin in vitro and in animal models. BMC Comp Altern Med 14: 15

24. Lorsuwannrat N, Piedrafita D, Chantree P, Sansri V, Sangkoomkrong S, et al. (2013) The in vitro anthelmintic effect of plumbagin on newly excysted and 4-week-old juvenile parasites of Fasciola gigantica. Exp Parasitol 136c: 5-13.

25. Dama LB, Jadhav BV (1997) Anthelmintic effect of juglone on mature and immature Hymenolepis nana in mice. Riv di Parasitol 2: 301-302.
26. Pinto AV, de Castro SL (2009) The trypanocidal activity of naphthoquinones: A review. Molecules 14: 4570-4590.

27. Bueding E, Peters L (1951) Effect of naphthoquinones on Schistosoma manson in vitro and in vivo. J Pharmacol Exp Ther 101: 210-229.

28. Pinto AV, Pinto MD, Gilbert B, Pellegrino J, Mello RT (1977) Schistosoma mansoni: blockage of cercarial skin penetration of chemical agents: 1. Naphthoquinones and derivatives. Trans R Soc Trop Hyg 71: 133-135

29. Lorsuwannarat $N$, Saowakon N, Ramasoota $P$, Wanichanon $C$, Sobhon $P$ (2013) The anthelmintic effect of plumbagin on Schistosoma mansoni. Exp Parasitol 133: 18-27.

30. Zhang SM, Coultas KA (2013) Identification of plumbagin and sanguinarine as effective chemotherapeutic agents for treatment of schistosomiasis. Int $J$ Parasitol 3: 28-34.

31. Lima NMF, dos Santos AF, Porfirio Z, Goulart MOF, Sant'Ana AEG (2002) Toxicity of lapachol and isolapachol and their potassium salts against Biophalaria glabarta, Schistosoma mansoni cercariae, Artmia salina and Tilapia nilotica. Acta Trop 83: 43-47.

32. Kapadia GJ, Azuine MA, Balasubramanian V, Sridhar R (2001) Aminonaphthoquinines. A novel class of compounds with potent antimalarial activity against Plasmodium falciparum. Pharmacol Res 43: 363-367.

33. Magalhães LG, Kapadia GJ, da Silva Tonuci LR, Caixeta SC, Parreira NA, et al. (2010) In vitro schistosomicidal effects of some pholoroglucinol derivatives from Dryopteris species against Schistosoma mansoni adult worms. Parasitol Res 106: 395-401.

34. Leyzama-Davila CD, Isaac-Marquez AP, Kapadia G, Owens K, Oghumu S, et al. (2012) Leishmanicidal activity of two naphthoquinones against Leishmania donovani. Biol Pharm Bull 35: 1761-1764.

35. Fisher LF, Hartwell JL (1935) The reaction of hydrazoic acid with naphthoquinones. J Am Chem Soc 57: 1483-1484.

36. Dalgliesh CE (1949) Naphthaoquinone antimalarials. Mannich bases derived from lawsone. J Am Chem Soc 71: 1697-1702.

37. Smithers SR, Terry RJ (1965) Infection of laboratory hosts with cercariae of Schistosoma mansoni and recovery of adult worms. Parasitol 55: 695-700.

38. Manneck T, Haggenmuller Y, Keiser J (2010) Morphological effects and tegumental alterations induced by mefloquine on schistosomal adult flukes on Schistosoma mansoni. Parasitol 137: 85-98.

39. Koné WM, Vargas M, Keiser J (2012) Anthelmintic activity of plants of Cote d'Ivoire for treating parasitic disease. Parasitol 110: 2351-2362.

40. Nwaka S, Hudson A (2006) Innovative lead discovery strategies for tropical diseases. Nat Rev Drug Discov 5: 941-955.

41. Thijessen HH, Vervoort LM, Schurqers LJ, Shearer MJ (2006) Menadione is a metabolite of oral vitamin K. Br J Nutr 95: 260-266.

42. Lanfranchi DA, Cesar-Rodo E, Bertrand B, Huang HH, Johann L, et al. (2012) Synthesis and biological evaluation of 1,4-naphthoquinones and quinoline5,8-diones as antimalarial and schistosomicidal agents. Org Biomol Chem 10 6375-6387.

43. Kuntz AN, Davioud-Charvet E, Sayed AA, Califf LL, Dessolin J, et al. (2007) Thioredoxin glutathione reductase from Schistosoma mansoni: An essential parasite enzyme and a key drug target. PLoS One 4: e264.

44. Kumar S, Gautam S, Sharma A (2013) Antimutagenic and antioxidant properties of plumbagin and other naphthoquinones. Mut Res 755: 30-41.

45. Ali A, Assimopoulou AN, Papageorgiou VP, Kolodziez H (2011) Structureantileishmanial activity relationship of naphthoquinones and dependency of the mode of action on the substitution patterns. Planta Med 77: 2003-2012.

46. Murakami K, Haneda M, Iwata S, Yoshino M (2010) Effect of hydroxy substituent on the prooxidant action of naphthoquinone compounds. Toxicol In Vitro 24: 905-909.

47. Huang HH, Rqouin C, Williams DL (2012) The redox biology of schistosome parasites and application for drug development. Curr Pharm Des 18: 35953611.

48. Johann L, Lafranchi DA, Davioud-Charvet E, Elhabiri M (2012) A physicobiochemical study on potential redox-cyclers as antimalarial and antischistosomal drugs. Curr Pharm Des 18: 3539-3566. 
Citation: Magalhães LG, Rao GS, Soares IAO, Badocco FR, Cunha WR, et al. (2014) Chemoprevention of Schistosomiasis: In vitro Antiparasitic Activity of Nineteen Plant-derived and Synthetic Simple Naphthoquinones and Naphthols against Schistosoma Mansoni Adult Worms. J Trop Dis 2: 146. doi: 10.4172/2329-891X.1000146

Page 7 of 7

49. Sayed AA, Cook SK, Williams DL (2006) Redox balance mechanisms in Schistosoma mansoni rely on peroxiredoxins and albumin and implicate peroxiredoxins as novel drug targets. J Biol Chem 281: 17001-17010.

50. Klaus V, Hartmann T, Gambini J, Graf P, Stahl W, et al. (2010)
1,4-Naphthoquinones as inducers of oxidative damage and stress signaling in HaCat human keratinocytes. Arch Biochem Biophys 496: 93-100.

51. Sharma N, Shukla AK, Das M, Dubey VK (2012) Evaluation of plumbagin and derivatives as potential modulators of redox thiol metabolism of Leishmania parasite. Parasitol Res 110: 341-348. 